controlling mesenchymal stem cell (MSC) growth (proliferation and apoptosis) and inhibiting myogenic differentiation under magnetic and non-magnetic conditions. The DAPT-loaded MNPs had an average hydrodynamic diameter of 351 d.nm Up to $40 \%$ of drug was released from MNPs within 48 hour rising to $65 \%$ after 1 week under magnetic conditions. The Notch ligand, Jagged1 increased Hey1 mRNA levels and promoted myogenic differentiation of MSCs in vitro by increasing SMC differentiation markers, myosin heavy chain 11 (Myh11) and calponin1 (CNN1) expression, respectively. This effect was significantly attenuated following treatment of cells with MNP's loaded with DAPT when compared to unloaded MNP's. Notch GSI -loaded magnetic nanoparticles are functional at targeting vascular stem cells in vitro.

\section{CHARACTERISATION OF RESIDENT MULTIPOTENT VASCULAR STEM CELLS (MVSCS) FROM SUSCEPTIBLE AND NON-SUSCEPTIBLE ARTERIOSCLEROTIC REGIONS OF THE MOUSE AORTA}

${ }^{1}$ Mariana Di Luca, ${ }^{1}$ Emma Fitzpatrick, ${ }^{2}$ Weimin Liu, ${ }^{2}$ David Morrow, ${ }^{2}$ Eileen M Redmond, ${ }^{1}$ Paul A Cahill*. 'Vascular Biology and Therapeutics Laboratory, School of Biotechnology Faculty of Science and Health, Dublin City University, Dublin 9, Ireland; ${ }^{2}$ Department of Surgery, University of Rochester Medical Centre, Rochester, NY, USA

\subsection{6/heartjnl-2018-SCF.25}

Vascular remodelling leading to arterial obstruction is a hallmark of arteriosclerosis and in-stent restenosis and is due in part to the accumulation of vascular smooth muscle (SMC)like cells within the vessel wall. The source of these vascular cells has been controversial with many studies providing compelling evidence for a putative role for stem cell-derived myogenic progeny. It is known that neuroectoderm-derived (NE) vascular regions (ascending aorta, aortic arch, carotid artery) are more susceptible to arteriosclerotic lesion formation in comparison with paraxial mesoderm-derived (PM) regions (descending and abdominal aorta, femoral artery). Our aim was to isolate and characterise stem cells from arterioscleroticsusceptible and non-susceptible regions and determine their differential responsiveness to discrete myogenic inductive stimuli. A population of myosin heavy chain (Myh11') negative, Sca1/S100ß/Nestin ${ }^{+}$multipotent vascular stem cells (MVSCs) was first shown to accumulate within the intima of murine carotid arteries following injury using e-GFP Sca1/S100 $\beta$ transgenic mice. Resident MVSCs were isolated from mouse aorta arch (susceptible) and descending aorta (non-susceptible) and grown in B27 supplemented maintenance media. Cells were characterised by fluorescent immunocytochemistry using stem (Sca1/S100ß/Nestin) and vascular SMC differentiation cell markers (Cnn1 and Myh11). MVSCs from both aortic arch and descending aorta were positive for stem cell markers but negative for Myh11 and Cnn1. Treatment of both cell populations with TGF- $\beta 1$ or the Notch ligand, Jagged-1 for 7 and 14 days promoted myogenic differentiation by increasing the number of cells expressing SMC markers concomitant with increased Myh11 and Cnn1 mRNA levels, respectively. Moreover, MVSCs from the NE susceptible regions significantly increased their expression of Myh11 in response to Jagged1, when compared to PM derived cells. We conclude that the aortic arch and descending aortic regions both house a neuroectoderm-derived Sca1/S100 $/ \mathrm{Nestin}^{+}{ }^{\text {stem }}$ cell population that responds to myogenic inductive stimulation in vitro. 